\title{
Papel da enfermagem na prevenção ao feminicídio
}

\author{
Role of nursing in the prevention of female \\ Papel de la enfermería en la prevención de la mujer
}

Alice Fonseca Pontes

ORCID: https://orcid.org/0000-0002-3291-5964 Universidade de Pernambuco, Brasil E-mail: alicepontes136@gmail.com

Beatriz Caetano da Silva

ORCID: https://orcid.org/0000-0003-4853-1663 Universidade de Pernambuco, Brasil

E-mail: beatrizcaetano20001@gmail.com

Natália Almeida Rodrigues

ORCID: https://orcid.org/0000-0002-6991-9168 Universidade de Pernambuco, Brasil

E-mail: natalia.almeida@upe.br

Mirela Ferreira Pessoa Deodoro

ORCID: https://orcid.org/0000-0001-9571-3828

Universidade de Pernambuco, Brasil

E-mail: mireladeodoro@gmail.com

Fernanda de Andrade Ribeiro

ORCID: https://orcid.org/0000-0001-9306-8167

Universidade de Pernambuco, Brasil

E-mail: fernanda.andraderibeiro@upe.br

Maria Eduarda Pereira de Souza

ORCID: https://orcid.org/0000-0003-2436-3706

Universidade de Pernambuco, Brasil

E-mail: eduarda.pereirasouza@upe.br

Marina Lopes de Melo Albuquerque

ORCID: https://orcid.org/0000-0002-1203-0843

Universidade de Pernambuco, Brasil

E-mail: marinalopesmelo@gmail.com

Marina Gomes de Oliveira Cabral

ORCID: https://orcid.org/0000-0002-1637-2648 Universidade de Pernambuco, Brasil

E-mail: marina.gomesc@upe.br

Rebeca Toledo Coelho

ORCID: https://orcid.org/0000-0003-2758-5179

Universidade de Pernambuco, Brasil

E-mail: rebecatnip@gmail.com

Nicole Hellen de Castro Barros

ORCID: https://orcid.org/0000-0002-6617-5765

Universidade de Pernambuco, Brasil

E-mail: nicole.barros@upe.br

Moama Natália Cruz da Silva

ORCID: https://orcid.org/0000-0002-1771-3248

Universidade de Pernambuco, Brasil E-mail: moama.cruz@upe.br

Jaynne Olívia da Silva Freitas

ORCID: https://orcid.org/0000-0001-5854-9547

Universidade de Pernambuco, Brasil

E-mail: jaynne.freitas@upe.br

Ingrid Samara Ramos Braga

ORCID: https://orcid.org/0000-0002-7934-509X

Universidade de Pernambuco, Brasil

E-mail: ingrid.samara@upe.br

Maria Clara Interaminense de Lucena

ORCID: https://orcid.org/0000-0003-0995-5121

Universidade de Pernambuco, Brasil E-mail: clara.ilucena@upe.br

Ângela Roberta Lessa de Andrade

ORCID: https://orcid.org/0000-0001-7753-675X

Universidade de Pernambuco, Brasil

E-mail: angelalessadeandrade@yahoo.com.br 


\title{
Resumo
}

Objetivo: O presente estudo busca entender o papel da enfermagem frente a prevenção do feminicídio, que no Brasil encontra-se cada vez mais frequente. Métodos: Revisão integrativa baseada na pergunta norteadora: Qual o papel da Enfermagem na prevenção ao feminicídio? As buscas foram realizadas nas bases de dados LILACS, BVS e SciELO, através dos descritores "Violência contra a mulher", "Direitos da mulher" e "Cuidados de Enfermagem", por meio dos booleanos $A N D$ e $O R$. Foram utilizados como critérios de inclusão artigos que estivessem disponíveis nos idiomas Português e Inglês, disponíveis na íntegra e datados nos últimos dez anos, devido à escassez em material que incluíssem a relação entre o feminicídio e os cuidados de enfermagem na saúde da mulher. Após análise, 16 publicações foram selecionadas para compor a discussão e apresentação desta revisão. Resultados: Com base na análise do material obtido nas buscas das bases de dados, ficou entendido a necessidade dos profissionais de enfermagem em incentivar as mulheres que estão ou passaram por situação de violências a realizarem o autocuidado e direcionar a rede de atendimento e assistência a essas vítimas, como forma de prevenção ao feminicídio. Sendo indispensável a orientação desses profissionais sobre o convívio social, visando o equilíbrio da saúde mental dessas mulheres. Conclusão: Apesar de ser um tema de total relevância na saúde pública atualmente, é preciso ampliar o debate acerca da violência de gênero dentro dos serviços de saúde, para que dessa forma, os profissionais de enfermagem possam compreender o fenômeno e lidar com as consequências da violência sofrida, sabendo que o cuidado prestado a essas mulheres é em sua maioria, realizado por essa classe profissional.

Palavras-chave: Cuidados de enfermagem; Direitos da mulher; Violência contra a mulher.

\begin{abstract}
Objective: This study seeks to understand the role of nursing in the prevention of femicide, which in Brazil is increasingly frequent. Methods: Integrative review based on the guiding question: What is the role of Nursing in preventing femicide?. Searches were carried out in the LILACS, BVS and SciELO databases, using the descriptors "Violence against women", "Women's rights" and "Nursing Care", through the Booleans AND and OR. Articles that were available in Portuguese and English, available in full and dated in the last ten years, were used as inclusion criteria, due to the scarcity of material that included the relationship between femicide and nursing care in women's health. After analysis, 16 publications were selected to compose the discussion and presentation of this review. Results: Based on the analysis of the material obtained in the searches of the databases, it was understood the need for nursing professionals to encourage women who are or have experienced violence to perform self-care and direct the service and assistance network to these victims, as a means of preventing femicide. The guidance of these professionals on social interaction is essential, aiming at balancing the mental health of these women. Conclusion: Despite being a topic of total relevance in public health today, it is necessary to broaden the debate on gender violence within health services, so that nursing professionals can understand the phenomenon and deal with the consequences of violence suffered, knowing that the care provided to these women is mostly performed by this professional class.
\end{abstract}

Keywords: Nursing care; Women rights; Violence against women.

\section{Resumen}

Objetivo: Este estudio busca comprender el papel de la enfermería en la prevención del feminicidio, que en Brasil es cada vez más frecuente. Métodos: Revisión integrativa a partir de la pregunta orientadora: ¿Cuál es el papel de la Enfermería en la prevención del feminicidio?. Las búsquedas se realizaron en las bases de datos LILACS, BVS y SciELO, utilizando los descriptores "Violencia contra la mujer", "Derechos de la mujer" y "Atención de enfermería", a través de los booleanos AND y OR. Los criterios de inclusión utilizados fueron artículos disponibles en portugués e inglés, disponibles íntegramente y fechados en los últimos diez años, debido a la escasez de material que incluyera la relación entre el femicidio y los cuidados de enfermería en la salud de la mujer. Después del análisis, se seleccionaron 16 publicaciones para componer la discusión y presentación de esta revisión. Resultados: A partir del análisis del material obtenido en las búsquedas de las bases de datos, se entendió la necesidad de que los profesionales de enfermería incentiven a las mujeres que son o han sufrido violencia a realizar el autocuidado y dirigir la red de servicio y asistencia a estas víctimas. como medio de prevención del feminicidio. La orientación de estos profesionales sobre la interacción social es fundamental, con el objetivo de equilibrar la salud mental de estas mujeres. Conclusión: A pesar de ser un tema de total relevancia en la salud pública actual, es necesario ampliar el debate sobre la violencia de género dentro de los servicios de salud, para que los profesionales de enfermería puedan comprender el fenómeno y enfrentar las consecuencias de la violencia sufrida, sabiendo que la atención brindada a estas mujeres se realiza principalmente por esta clase profesional.

Palabras clave: Cuidado de enfermera; Derechos de las mujeres; Violencia contra las mujeres.

\section{Introdução}

A violência contra a mulher é um problema de saúde pública que repercute uma longa discussão sobre as medidas de prevenção ao feminicídio e os motivos que o acometem. Segundo Moura (2020), a violência é uma violação crítica dos direitos humanos, que mexe de forma dolorosa na vida da mulher. Esse fenômeno que ocorre sob tantos contextos, aborda toda e qualquer 
forma de violência que muitas vezes, pode levar ao assasinato da mulher, onde fica entendido como violência tudo aquilo que gera dano, muitas vezes inconversíveis, deixando múltiplas marcas físicas, sexuais ou mentais, privando-lhes do livre desfrute da vida (Honnef, et al. 2017).

No Brasil, a violência de gênero se encontra cada vez mais frequente. Mulheres estão sendo mortas apenas por serem mulheres (Souza, 2020). Esse mal que vem muitas vezes em forma de maus tratos, abuso sexual, ameaças ou pressão psicológica, não olha classe social, cor da pele ou idade (Netto, et al. 2019). A vítima muitas vezes é violentada por pessoas próximas, sendo elas companheiros, vizinhos ou até os próprios familiares, o que engata em diversos casos a dependência emocional ao seu agressor (Rabelo, et al. 2019).

A privação social contra a mulher já existe há anos. Com o feminismo, muitas revoluções na vida das mulheres iniciaram-se, como direito de participação na política e de escolher seu próprio destino (Martins, et al. 2020). Apesar disso, segundo Benevides e Nogueira (2020, p. 20), o Brasil teve sua colocação de $1^{\circ}$ lugar no mundo em assassinatos de pessoas trans desde o ano de 2008, com um total de 175 registros de pessoas assassinadas por expressarem a identidade de gênero feminino, presente no Mapa de assassinatos do ano de 2020 (Benevides; Nogueira, 2020, p. 9). Esse fenômeno violento, é uma urgência aos profissionais de enfermagem inseridos no sistema de saúde, para buscarem meios de cuidados a essas vítimas, direcionandoas à denúncia e à busca pelo cuidado, como forma de prevenção ao feminicídio.

Ainda assim, para mulheres negras e transexuais, muitas barreiras são mais espessas, como a da sobrevivência. O Atlas da Violência no Brasil, mostra que entre os anos de 2008 e 2018, a violência contra a mulher negra teve um aumento de 12,4\%, enquanto a taxa de violência contra mulheres não pretas reduziu 11,7\%. Nos dados atuais, dos anos de 2017 a 2018 , houve uma redução de 8,4\% nos homicídios contra a mulher, mas ainda assim, com uma discrepância racial: a taxa de homicídios de mulheres não negras reduziu 12,3\%, e a de mulheres negras reduziu cerca de 7,2\% (Ipea, 2020).

A Lei n ${ }^{\circ}$ 11.340, que entrou em vigor em 07 de Agosto de 2006, é conhecida como Lei Maria da Penha, devido à luta de Maria da Penha Fernandes Maia, que buscou justiça por 19 anos e 6 meses após sofrer de diversas agressões pelo marido, que teve danos irreversíveis (Brasil, 2006). Ela tornou-se símbolo de luta e liberdade, com o objetivo de proteger mulheres de atos violentos, trazendo assistência, amparo e garantindo proteção (Cardoso; Brito, 2015). Com base no artigo 226 da Constituição Federal, instituída a Conferência Interamericana, fica evidente a urgência de mecanismos para prevenir e proteger a mulher contra todas as formas de violência. A criação de medidas para fornecer assistência e proteção às vítimas de violência dispõe sobre a criação dos Juizados de Violência Doméstica e Familiar contra a Mulher (Brasil, 2006).

No Brasil, em 2007, foi instaurada a Lei nº 11.489, que declara apoio ao Dia Nacional de Mobilização dos Homens pelo Fim da Violência Contra as Mulheres. Segundo dados do Fórum Brasileiro de Segurança Pública, no Brasil, durante o período da pandemia da Covid-19, os registros de feminicídio aumentaram 22,2\% e o canal de denúncia Ligue 180, Central Nacional de Atendimento à Mulher, registrou aumento de 24\% (Fbsp, 2020). Com a Lei no 13.104 de 9 de Março de 2015, ficou em vigor, de acordo com o Código Penal, que o feminicídio é uma circunstância do crime de homicídio (Brasil, 2015). Com essa inclusão, foram notificados o aumento de 929 casos em 2016 para 1.326 no ano de 2019 (Fbsp, 2020).

Com a Campanha Sinal Vermelho contra a Violência Doméstica, foi decretada a Lei no 14.188/2021, uma medida de combate a violência contra a mulher (Brasil, 2021). O apoio às mulheres ou testemunhas de violência contra a mulher pode ser feito pelo Ligue 180, suporte de denúncia que funciona 24 por dia e de forma gratuita, pelo qual a mulher consegue ser encaminhada aos órgãos competentes de atendimento (Brasil, 2021). Esse serviço disponível em todo território brasileiro, provê informações sobre os locais mais próximos para melhor suporte: Centros de Referências, Delegacias de Atendimento à Mulher (DEAM), Núcleos Integrados de Atendimento às Mulheres, Defensorias Públicas e outros (Brasil, 2021).

O Sistema Único de Saúde (SUS) oferta diariamente atendimento de forma integral às mulheres vítimas de violência (Acosta, et al. 2017). O profissional da Enfermagem é um dos muitos profissionais presentes na equipe multiprofissional de 
saúde, que deve trabalhar de forma a realizar um atendimento dinâmico, humanizado e holístico (Arboit, et al. 2017). A Enfermagem como um todo, busca aplicar a integralidade em saúde, uma das diretrizes do SUS, como forma de assistência preventiva por meio de ações de educação em saúde, cuidados individuais e coletivos (Silva, et al. 2021).

A carência de atenção em saúde às vítimas, mostra cada dia que os profissionais de enfermagem encontram-se despreparados no atendimento imediato em casos de violência contra a mulher (Silva, et al. 2021). Assim, a assistência de enfermagem acaba tornando-se paliativa, sendo restrita aos cuidados de lesões (Colimoide, et al. 2017).

Ficou evidente a necessidade de urgência na criação de novas medidas para combater a morte de mulheres vítimas de violência contra a mulher. Por meio de atenção integral à saúde e suporte de educação continuada a profissionais de Enfermagem, uma ponte de assistência deve ser criada como uma forma de conter o feminicídio, uma violência de gênero que cresce todos os dias (Arboit, et al. 2017).

A partir desta contextualização, este trabalho objetiva entender, por meio de uma revisão integrativa, como a atuação dos profissionais de enfermagem podem contribuir para prevenir casos de feminicídio, visto que, comumente, estes atendem vítimas de violência que precedem o feminicídio.

\section{Metodologia}

Este estudo é uma revisão integrativa, que consiste na abordagem metodológica mais ampla entre as revisões de literatura. Este tipo de revisão permite a integração de estudos experimentais e não experimentais para que seja possível a compreensão completa do fenômeno estudado (De Souza, et al. 2017, p. 18). Segundo Andrade, et al. (2017) a metodologia tem a finalidade de gerar síntese de como os resultados foram adquiridos nas pesquisas sobre um determinado tema, de forma sistemática e ordenada, concedendo informações amplas.

A revisão integrativa é formada pelo total de seis fases: 1) produção de uma pergunta norteadora: vai definir os artigos que serão incluídos na pesquisa; 2) amostragem na literatura: busca nas base de dados; 3) coleta de dados: obtenção de dados dos artigos selecionados; 4) análise dos estudos incluídos: estratégia rigorosa na avaliação das características de cada estudo; 5) discussão dos resultados: comparação dos dados evidenciados; 6): apresentação dos resultados: deve ser clara para que o leitor avalie criticamente os resultados (De Souza, et al. 2017, p. 18).

Ademais, fica evidente que a diversidade na composição da amostragem de uma revisão integrativa fortalece o conjunto de conceitos, teoria e problemas voltados aos cuidados de saúde, tornando-se um grande conjunto de alta relevância para a Enfermagem (Andrade, et al. 2017). Pensando assim, foi formulada a seguinte pergunta norteadora: Qual o papel da Enfermagem na prevenção ao feminicídio?

Com isso, as buscas foram realizadas nas bases de dados online Literatura Latino-Americana e do Caribe em Ciências da Saúde (LILACS), Biblioteca Virtual em Saúde (BVS) e Scientific Electronic Library Online (SciELO) através dos descritores "Violência contra a mulher", "Direitos da mulher" e "Cuidados de Enfermagem", por meio dos booleanos AND e OR. Foram utilizados como critérios de inclusão, artigos que estivessem disponíveis no idioma Português e Inglês disponíveis na íntegra, datados nos últimos dez anos e que incluíssem a relação entre o feminicídio e os cuidados de enfermagem na saúde da mulher.

Esse recorte temporal foi preciso para melhor compreensão da temática, devido à escassez de material voltado à atuação da enfermagem frente à prevenção do feminicídio.

Logo, foram encontradas 225 publicações, nas quais analisou-se os títulos e resumos, como também, os critérios de inclusão e exclusão proposto. Em seguida, n=38 publicações foram pré-selecionadas para ser realizada a leitura na íntegra com o intuito de analisar criticamente os resultados obtidos. Ao todo, $n=22$ publicações foram excluídas por não atenderem aos critérios de inclusão do estudo. Após o processo de análise, n=16 publicações foram selecionadas para compor os resultados e apresentação desta revisão integrativa. Abaixo segue a Figura 1 mostrando o esquema de seleção por meio de um fluxograma: 
Figura 1: Fluxograma sobre a busca das publicações científicas e as bases de dados. Recife, Pernambuco, Brasil.

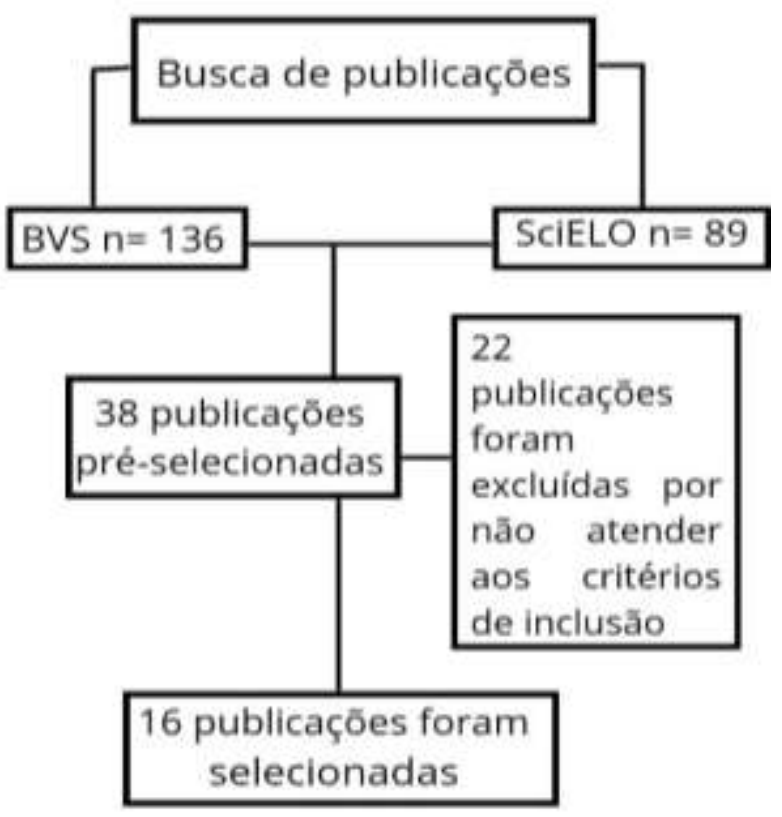

Fonte: Pontes, et al. (2021).

\section{Resultados}

A amostra final desta revisão foi constituída por dezesseis artigos científicos, utilizando como critério de inclusão os que estivessem disponíveis no idioma Português, datados nos últimos 10 anos e que abordassem a relação entre a enfermagem e a prevenção ao feminicídio. Buscou-se trabalhos nas bases de dados Literatura Latino-Americana e do Caribe em Ciências da Saúde (LILACS), Biblioteca Virtual em Saúde (BVS) e Scientific Electronic Library Online (SciELO).

Ademais, nesta revisão foram incluídos dezesseis artigos científicos, os quais estavam disponíveis para leitura na íntegra nas seguintes bases de dados: LILACS (0), BVS (8), e SciELO (8). Nesse contexto, pode-se elucidar os resultados adquiridos com os artigos utilizados e de maior relevância, aplicando-se a um o Quadro 1.

Quadro 1. Artigos levantados nas bases de dados BVS e SciELO. Recife, Pernambuco, Brasil.

\begin{tabular}{|c|c|c|c|}
\hline Título & Autores & Periódicos & Considerações/ Temática \\
\hline $\begin{array}{l}\text { Incidência da Violência } \\
\text { contra a Mulher e a Lei do } \\
\text { Feminicídio. }\end{array}$ & $\begin{array}{l}\text { Domingas Pereira Rabelo; } \\
\text { Kátia Costa dos Santos; } \\
\text { Elisângela de Andrade Aoyama. }\end{array}$ & $\begin{array}{l}\text { ReBIS [Internet]. 2019; } \\
\text { 1(4):71-6. }\end{array}$ & $\begin{array}{l}\text { Busca identificar a incidência da violência } \\
\text { contra a mulher e a lei do Feminicídio. }\end{array}$ \\
\hline $\begin{array}{l}\text { Representações sociais da } \\
\text { violência doméstica em } \\
\text { cenários rurais para } \\
\text { mulheres e homens }\end{array}$ & $\begin{array}{l}\text { Fernanda Honnef; Marta Cocco } \\
\text { da Costa; Jaqueline Arboit; } \\
\text { Ethel Bastos da Silva; Karoline } \\
\text { Ardenghi Marques. }\end{array}$ & $\begin{array}{l}\text { Acta Paul Enferm } 30 \text { (4), } \\
\text { Jul-Aug, 2017. São Paulo } \\
\text { - SP, Brasil. }\end{array}$ & $\begin{array}{l}\text { Busca compreender as representações sociais } \\
\text { da violência doméstica em cenários rurais, na } \\
\text { perspectiva de mulheres e homens residentes } \\
\text { nesses contextos. }\end{array}$ \\
\hline $\begin{array}{l}\text { Planejamento } \\
\text { implementação do curso } \\
\text { Sexual Assault Nurse } \\
\text { Examiner } \\
\text { para assistir vítimas de } \\
\text { violência sexual: um relato } \\
\text { de experiência }\end{array}$ & $\begin{array}{l}\text { Juliana de Oliveira Musse Silva; } \\
\text { Eileen Marie Allen; Isle } \\
\text { Polonko; Karen Beatriz Silva; } \\
\text { Rita de Cassia Silva; Rafael } \\
\text { Braga Esteves. }\end{array}$ & $\begin{array}{l}\text { Rev Esc Enferm USP } \\
\text { 2021;55:e } 03739\end{array}$ & $\begin{array}{l}\text { Explica as experiências dos profissionais de } \\
\text { saúde em relação ao atendimento de vitimas de } \\
\text { violência sexual. }\end{array}$ \\
\hline $\begin{array}{l}\text { Integralidade na } \\
\text { perspectiva de enfermeiros }\end{array}$ & $\begin{array}{l}\text { Fabiana da Penha Colimoide; } \\
\text { Maria Dyrce Dias Meira; Gina }\end{array}$ & $\begin{array}{l}\text { Rev. Bioét. } 25 \text { (3), Sep- } \\
\text { Dec } 2017\end{array}$ & $\begin{array}{l}\text { Explica a percepção de enfermeiros sobre a } \\
\text { integralidade do ser humano na prática do }\end{array}$ \\
\hline
\end{tabular}




\begin{tabular}{|c|c|c|c|}
\hline $\begin{array}{l}\text { da Estratégia Saúde da } \\
\text { Família. }\end{array}$ & $\begin{array}{l}\text { Andrade Abdala; Sarah Lidiane } \\
\text { Santos da Silva Oliveira. }\end{array}$ & & cuidado de enfermagem. \\
\hline $\begin{array}{l}\text { Aspectos éticos e legais no } \\
\text { cuidado de enfermagem às } \\
\text { vítimas de violência } \\
\text { doméstica. }\end{array}$ & $\begin{array}{l}\text { Daniele Ferreira Acosta; Vera } \\
\text { Lúcia de Oliveira Gomes; } \\
\text { Denize Cristina de Oliveira; } \\
\text { Giovana Calcagno Gomes; } \\
\text { Adriana Dora da Fonseca. }\end{array}$ & $\begin{array}{l}\text { Texto contexto - enferm. } \\
26(3), 2017\end{array}$ & $\begin{array}{l}\text { Traz uma análise sobre o conhecimento de } \\
\text { enfermeiras hospitalares sobre os aspectos } \\
\text { éticos e legais no cuidado de enfermagem às } \\
\text { vítimas de violência doméstica. }\end{array}$ \\
\hline $\begin{array}{l}\text { Health care for women in } \\
\text { situations of violence: } \\
\text { discoordination of network } \\
\text { professionals. }\end{array}$ & $\begin{array}{l}\text { Jaqueline Arboit; Stela Maris de } \\
\text { Mello Padoin; Letícia Becker } \\
\text { Vieira; Cristiane Cardoso de } \\
\text { Paula; Marta Cocco da Costa; } \\
\text { Laura Ferreira Cortes. }\end{array}$ & $\begin{array}{l}\text { Rev. esc. enferm. USP 51, } \\
2017\end{array}$ & $\begin{array}{l}\text { Trata-se de explicar as concepções e ações dos } \\
\text { profissionais de saúde na rede de atenção à } \\
\text { mulher em situação de violência. }\end{array}$ \\
\hline $\begin{array}{l}\text { Atuação da enfermagem na } \\
\text { conservação da saúde de } \\
\text { mulheres em situação de } \\
\text { violência. }\end{array}$ & $\begin{array}{l}\text { Leônidas de Albuquerque } \\
\text { Netto; Eric Rosa Pereira; Joyce } \\
\text { Martins Arimatea Branco } \\
\text { Tavares; Dennis de Carvalho } \\
\text { Ferreira; Priscilla Valladares } \\
\text { Broca. }\end{array}$ & $\begin{array}{l}\text { Rev. Mineira de } \\
\text { Enfermagem, Volume: } \\
\text { 22:e-1149. }\end{array}$ & $\begin{array}{l}\text { Analisar pela ótica da Teoria de Enfermagem } \\
\text { de Levine, o atendimento da enfermagem às } \\
\text { mulheres que sofreram violência. }\end{array}$ \\
\hline $\begin{array}{lr}\text { Acolhimento } & \mathrm{e} \\
\text { acompanhamento } & \mathrm{de} \\
\text { mulheres vítimas de } & \\
\text { violência sexual: proposta } \\
\text { de ferramenta de apoio. }\end{array}$ & Leticia de Souza Moura. & $\begin{array}{l}\text { Dissertação de mestrado. } \\
\text { Curitiba, Paraná, Brasil, } \\
2020 .\end{array}$ & $\begin{array}{l}\text { Tabalha as ferramentas de apoio e acolhimento } \\
\text { no cuidado de mulheres vitima de violência } \\
\text { sexual, atendidas no Pronto Atendimento de } \\
\text { Ginecologia e Obstetrícia. }\end{array}$ \\
\hline $\begin{array}{l}\text { Educação permanente em } \\
\text { saúde: desenvolvimento de } \\
\text { competências profissionais } \\
\text { na atenção às mulheres em } \\
\text { situação de violência. }\end{array}$ & $\begin{array}{l}\text { Mari Aparecida Rocha de } \\
\text { Souza. }\end{array}$ & $\begin{array}{l}\text { Dissertação de mestrado. } \\
\text { Curitiba, Paraná, Brasil, } \\
2020 .\end{array}$ & $\begin{array}{l}\text { A pesquisa aborda o desenvolvimento de } \\
\text { competências profissionais para a promoção de } \\
\text { ações articuladas, com foco nas necessidades } \\
\text { dessas mulheres. Tendo como objetivo } \\
\text { estruturar um modelo de Educação Permanente } \\
\text { em Saúde, para o desenvolvimento de } \\
\text { competências dos profissionais de uma rede } \\
\text { intersetorial de atenção à mulher em situação } \\
\text { de violência. }\end{array}$ \\
\hline $\begin{array}{l}\text { Possíveis impasses da } \text { Lei } \\
\text { Maria da Penha à } \\
\text { convivência parental. }\end{array}$ & $\begin{array}{l}\text { Fernanda Simplício Cardoso; } \\
\text { Leila Maria Torraca de Brito. }\end{array}$ & $\begin{array}{l}\text { Estudos e Pesquisas em } \\
\text { Psicologia, Rio de Janeiro } \\
\text { v. } 15 \text { n. } 2 \text { p. } 529-546 . \\
2015 .\end{array}$ & $\begin{array}{l}\text { Buscou discorrer sobre a temática da } \\
\text { convivência parental, a partir dos pressupostos } \\
\text { teóricos da Psicologia Jurídica, em sua } \\
\text { interface com o Direito de Família. A questão } \\
\text { problematizada são os possíveis } \\
\text { desdobramentos da lei } 11.340 / 2006 \text {, conhecida } \\
\text { como Lei Maria da Penha, na convivência dos } \\
\text { filhos com o pai, suposto autor da agressão. }\end{array}$ \\
\hline $\begin{array}{l}\text { A metodologia de revisão } \\
\text { integrativa da literatura em } \\
\text { enfermagem. }\end{array}$ & $\begin{array}{l}\text { Luís Manuel Mota de Sousa; } \\
\text { Cristina Maria Alves Marques- } \\
\text { Vieira; Sandy Silva Pedro } \\
\text { Severino; Ana Vanessa } \\
\text { Antunes. }\end{array}$ & $\begin{array}{l}\text { Rev. Investigação em } \\
\text { Enf. n 21. } 2^{\mathrm{a}} \text { série - } \\
\text { Novembro } 2017\end{array}$ & $\begin{array}{l}\text { Almejou apresentar os conceitos gerais e as } \\
\text { etapas para a elaboração de uma revisão } \\
\text { integrativa da literatura }\end{array}$ \\
\hline $\begin{array}{l}\text { Vítimas de violência sexual } \\
\text { atendidas em um serviço de } \\
\text { referência. }\end{array}$ & $\begin{array}{l}\text { Tatiane Herreira Trigueiro; } \\
\text { Miriam Aparecida Barbosa } \\
\text { Merighi; Adeli Regina } \\
\text { Prizybicien de Medeiros; Cléa } \\
\text { Elisa Lopes Ribeiro; Nely } \\
\text { Dayse Santos da Mata; Maria } \\
\text { Cristina Pinto de Jesus. }\end{array}$ & $\begin{array}{l}\text { Cogitare } \quad \text { Enferm. } \\
\text { 20(2):249-56. } 2015 .\end{array}$ & $\begin{array}{l}\text { Visa conhecer aspectos da violência sexual e } \\
\text { adesão das vítimas ao seguimento } \\
\text { ambulatorial. }\end{array}$ \\
\hline $\begin{array}{l}\text { Cuidado à mulher em } \\
\text { situação de violência } \\
\text { doméstica: } \\
\text { de representações } \\
\text { hospitalares. }\end{array}$ & $\begin{array}{l}\text { Daniele Ferreira Acosta; Vera } \\
\text { Lúcia de Oliveira Gomes; } \\
\text { Denize Cristina Oliveira; Sergio } \\
\text { Corrêa Marques; Giovana } \\
\text { Calcagno Gomes. }\end{array}$ & Rev. Eletr. Enf. 2015. & $\begin{array}{l}\text { Busca analisar a estrutura e os conteúdos das } \\
\text { representações sociais de enfermeiras(os) } \\
\text { acerca do cuidado à vítima de violência } \\
\text { doméstica. }\end{array}$ \\
\hline
\end{tabular}




\begin{tabular}{|c|c|c|c|}
\hline $\begin{array}{l}\text { Violência contra a mulher: } \\
\text { como os profissionais na } \\
\text { atenção primária à saúde } \\
\text { estão enfrentando esta } \\
\text { realidade? }\end{array}$ & $\begin{array}{l}\text { Silvana Cavalcanti dos Santos; } \\
\text { Patricia de Albuquerque Barros; } \\
\text { Rafaella França de Araújo } \\
\text { Delgado; Luiza Vanessa de } \\
\text { Lima Silva; Valdirene P. da } \\
\text { Silva Carvalho; Ana Carla Silva } \\
\text { Alexandre. }\end{array}$ & $\begin{array}{l}\text { Rev. Saúde e Pesquisa, v. } \\
\text { 11, n. 2, p. 359-368. } 2018 .\end{array}$ & $\begin{array}{l}\text { Tentar identificar as formas de assistência } \\
\text { prestada pelos profissionais da atenção } \\
\text { primária à mulher vítima de violência no } \\
\text { município de Buíque (PE). }\end{array}$ \\
\hline $\begin{array}{l}\text { O estudo de caso como } \\
\text { método de pesquisa em } \\
\text { enfermagem: uma revisão } \\
\text { integrativa. }\end{array}$ & $\begin{array}{l}\text { Selma Regina de Andrade; } \\
\text { Andriela Backes Ruoff; Talita } \\
\text { Piccoli; Márcia Danieli Schmitt; } \\
\text { Alexandra Ferreira; Ana } \\
\text { Cristina Ammon Xavier. }\end{array}$ & $\begin{array}{l}\text { Texto contexto - enferm. } \\
26 \text { (4), } 2017\end{array}$ & $\begin{array}{l}\text { Busca mostrar a aplicação do estudo de caso } \\
\text { como método de pesquisa pela enfermagem } \\
\text { nas publicações científicas nacionais e } \\
\text { internacionais. }\end{array}$ \\
\hline $\begin{array}{l}\mathrm{O} \quad \text { efeito das } \\
\text { microagressões raciais de } \\
\text { gênero na saúde mental de } \\
\text { mulheres negras }\end{array}$ & $\begin{array}{l}\text { Tafnes Varela Martins; Tiago } \\
\text { Jessé Souza de Lima; Walberto } \\
\text { Silva Santos; Tafnes Varela } \\
\text { Martins; Tiago Jessé Souza de } \\
\text { Lima; Walberto Silva Santos. }\end{array}$ & $\begin{array}{l}\text { Ciênc. saúde coletiva } 25 \\
\text { (7), Jul } 2020\end{array}$ & $\begin{array}{l}\text { Avalia os impactos de violência de gênero } \\
\text { contra a mulher e a saúde mental de mulheres } \\
\text { negras. }\end{array}$ \\
\hline
\end{tabular}

Fonte: Pontes, et al. (2021).

Com base na análise do material obtido nas buscas das bases de dados, ficou entendido a necessidade dos profissionais de enfermagem em incentivar as mulheres que estão ou passaram por situação de violências a realizar o autocuidado e direcionar a rede de atendimento e assistência à essas vítimas, como forma de prevenção ao feminicídio. Sendo indispensável a orientação desses profissionais sobre o convívio social, visando o equilíbrio da saúde mental dessas mulheres. Nota-se então, que o SUS possui extrema importância na problemática da violência contra mulher e diante disso, a enfermagem, como atuante desse sistema, deve ter a capacidade de reconhecer o problema, acolher a vítima, referir para a rede especializada (a fim de garantir os direitos das mulheres, proporcionando um atendimento com ações humanizadas e eticamente seguras), assim como, deve também incentivar a promoção de políticas públicas de saúde e da legislação vigente, com o intuito de propiciar segurança para as mulheres.

\section{Impactos na saúde mental em mulheres vítimas de violência}

Os impactos instaurados na saúde mental da mulher, tem base em cinco ramos centrais causadores de danos, de acordo com Netto, et al. (2020): falta de energia, desestruturação pessoal e social em vítimas de violência, seguida da falta do autocuidado, levando à sua exclusão do convívio com familiar e com pessoas próximas, acarretando em transtornos psicológicos. Ainda segundo Netto, et al. (2020), a conservação de cuidados em saúde parte fortemente dos profissionais da enfermagem, buscando uma atenção integral.

Segundo Netto, et al. (2020), o bem-estar mental é algo que vai além do que é possível ver, transcendendo na autoestima da mulher, possuindo dimensões emocionais, psicológicas, fisiológicas e cultural, podendo ser divida em 5 categorias, presentes na Figura 2: 
Figura 2. Os quatro impactos instaurados na saúde mental da mulher. Recife, Pernambuco, Brasil.

\section{Ausência de energia}

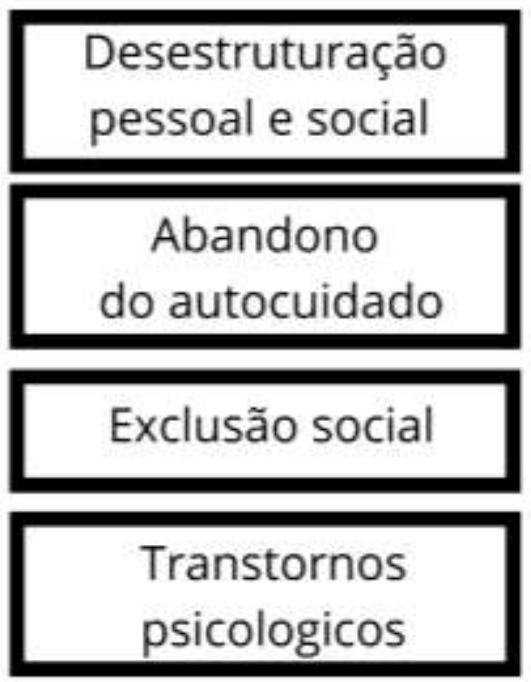

Fonte: Pontes, et al. (2021).

\section{Atuação da enfermagem na prevenção ao feminicídio}

O Enfermeiro possui uma função muito relevante na assistência às mulheres vítimas de violência, pois atua diretamente no acolhimento, atendimento e reabilitação dessas usuárias do SUS. O objetivo do cuidado de enfermagem nesses casos, é promover a recuperação das clientes, por meio de condições adequadas de trabalho e conhecimentos específicos do Protocolo De Atenção Básica Para Saúde Da Mulher.

$\mathrm{O}$ atendimento e assistência à mulher deve ser de extrema importância para a equipe de enfermagem pois os eventos vivenciados pelas usuárias podem acarretar em agravos psicológicos e físicos de grande relevância para o seu quadro clínico, como: o abandono do autocuidado; a exclusão social; a desestruturação pessoal, familiar e social, entre outros agravos. Sendo necessário, que a atuação da enfermagem seja pautada na associação de ações de educação em saúde, como forma de prevenção e a própria reabilitação e assistência das vítimas após acontecido o fato.

Os artigos encontrados demonstram que os enfermeiros possuem conhecimento para realizar um atendimento eficaz, através da escuta qualificada, na qual, deverão prestar bastante atenção em suas queixas e identificar marcas ou expressões que revelem possíveis sinais de violência. Também é importante que o profissional estabeleça um nível de confiança com essa mulher, dentro do serviço de saúde, pois facilita as ações preventivas, o registro, o encaminhamento e acompanhamento dos casos, potencializando a assistência.

Além disso, cabe ao profissional de enfermagem proporcionar suporte e confiança a essas vítimas, mobilizando recursos sociais e familiares; além de auxiliá-la em como poderá enfrentar a situação, ajudando dessa maneira, a retirá-la desse ciclo de violência. Ele deve conscientizar as vítimas de seus direitos, para auxiliar-lhes a tomar decisões positivas e seguras, com a intenção de quebrar o ciclo da violência.

Assim como, é importante e necessário que a equipe de enfermagem possa dialogar com essa mulher sobre o empoderamento, autonomia e liberdade financeira, de forma que ela possa tornar-se independente e segura, dando-lhe o apoio necessário para que ela fortaleça os laços familiares e sociais. 
Cabe também ao enfermeiro, intermediar a interação entre as Equipes da Estratégia de Saúde da Família (ESF) e do Núcleo de Apoio à Saúde da Família (NASF), serviços aos quais a mulher pode buscar e obter o auxílio necessário para enfrentamento de sua condição de vulnerabilidade (Brasil, 2016).

Ainda assim, se tem a necessidade de estudos mais aprofundados visando sanar lacunas acerca da atuação dos profissionais de enfermagem frente à prevenção do feminicídio e aos cuidados de vítimas de violência contra a mulher, pensando na oferta de uma atenção integral à saúde da mulher.

\section{Discussão}

A problemática da violência contra a mulher é de extrema importância no âmbito do SUS, que tenta por meio de seus profissionais perceber e notificar os casos, além de atender as demandas das vítimas, como forma de devolver-lhes a autonomia. A violência doméstica e intrafamiliar contra a mulher, configura-se como uma violação dos seus direitos fundamentais e constitui um sério problema de saúde pública, sendo uma das principais causas de morbidade e mortalidade feminina (Brasil, 2016). Segundo o Ministério da Saúde (2016), esta violência ocorre principalmente entre pessoas da mesma parentela e parceiros íntimos. As violências podem ser divididas em três categorias quanto ao tipo: auto-infligida, interpessoal e coletiva (Pernambuco, 2010).

Além disso, para os casos de violência contra a mulher, pode ser classificado em cinco tipos de acordo com a natureza: moral, patrimonial, psicológica, sexual e física. Essa classificação (Quadro 2) está presente na Lei nº 11.340 de 2006, conhecida como Lei Maria da Penha, a qual criou mecanismos para prevenir e punir este tipo de violência, anteriormente bastante subnotificado, devido à falta de redes de apoio à mulher vítima de violência (Brasil, 2006).

Quadro 2. Os cinco tipos de violência classificados quanto a natureza de acordo com a Lei no 11.340/2006 (Lei Maria da Penha). Recife, Pernambuco, Brasil. (Brasil, 2006).

\begin{tabular}{|l|l|}
\hline Violência Moral & Atitude que configura calúnia, difamação e injúria. \\
\hline Violência & $\begin{array}{l}\text { Conduta que configure subtração, retenção, destruição parcial ou total de seus objetos, } \\
\text { instrumentos de trabalho, documentos e recursos destinados a satisfazer suas necessidades. }\end{array}$ \\
\hline $\begin{array}{l}\text { Violência } \\
\text { Psicológica }\end{array}$ & $\begin{array}{l}\text { Condutas que causem dano emocional e diminuição da autoestima, que prejudicam o seu } \\
\text { pleno desenvolvimento, visando controlar suas ações, comportamentos, crenças e decisões. } \\
\text { Mediante ameaça, constrangimento, humilhação, manipulação, vigilância. }\end{array}$ \\
\hline Sexual & $\begin{array}{l}\text { Entendida como aquela em que a mulher se sinta constrangida a presenciar, manter ou } \\
\text { participar de relação sexual não desejada, por meio de intimidação, ameaça, coação, uso da } \\
\text { força, chantagem, suborno ou manipulação, atitudes que limitam ou anulam o exercício dos } \\
\text { seus direitos sexuais e reprodutivos. }\end{array}$ \\
\hline $\begin{array}{l}\text { Violência } \\
\text { Física }\end{array}$ & $\begin{array}{l}\text { Conduta que ofenda a integridade ou saúde corporal da vítima. Tapas, chutes, torções, } \\
\text { empurrões, estrangulamento, queimaduras, entre outras lesões. }\end{array}$ \\
\hline
\end{tabular}

Fonte: Brasil (2006).

Sabe-se ainda que o número de mulheres negras vítimas de violência é maior que o quantitativo para mulheres brancas (Ipea, 2020). Atualmente ainda existe uma grande incógnita quanto a demanda da mulher negra em relação às instituições e 
profissionais de saúde no que se refere à sua sexualidade, saúde reprodutiva e situações de violência. Essa incognita vem tornando-se uma discussão cada vez maior, explicada pela discriminação racial institucional que ocorre com essas mulheres (Carrijo, 2020). Por isso, há uma necessidade iminente de realizar maiores pesquisas sobre a situação e prevalência da violência em mulheres negras a partir de um referencial humanista.

Outro grupo vulnerável entre as mulheres, são as transexuais e transgêneras, as quais, tornam-se vítimas da violência doméstica devido ao histórico de machismo e transfobia enraizados no comportamento social, que corroboram com esse ciclo de dominação e violência constantemente presente no contexto social do Brasil (Zabala, 2016). Muitas vezes essas mulheres são violentadas por sua família, que as exclui da esfera social, do ambiente escolar e familiar. Essas mulheres tornam-se invisíveis socialmente e negligenciadas pelo Estado, fatos que contribuem para a perpetuação da violência para com este grupo, pois percebe-se a ausência de prevenção e combate à violência contra a população trans (Zabala, 2016).

Além disso, como agravante para este problema, o cenário da pandemia da COVID-19 ocasionou o relevante aumento dos casos de violência contra a mulher. A situação de calamidade pública reprimiu ainda mais a capacidade da mulher de evitar ou fugir dos potenciais conflitos com o agressor, aumentando os casos notificados durante este período (Okabayashi, et al. 2020). Ademais, a abordagem de acolhimento a essas vítimas durante esse período caótico de pandemia e a sobrecarga profissional da enfermagem, viu-se a necessidade imediata de novas abordagem de políticas públicas na prevenção ao feminicídio, visando atenção integral dessas mulheres.

No dia 28 de julho de 2021, foi promulgada a Lei $n^{\circ} 14.188$, que define a campanha de cooperação Sinal Vermelho, como uma das medidas de enfrentamento à violência doméstica e familiar contra a mulher, previstas na Lei Maria da Penha (Brasil, 2021). Esta lei, visa alterar o código penal (Lei n 2.848/1940) para modificar e tornar mais severa a penalidade por lesão corporal simples cometida contra a mulher, além de criar um tipo penal específico para o crime de violência psicológica (Brasil, 2021). A ideia central é que uma mulher consiga pedir ajuda em estabelecimentos públicos e privados, por meio de um " $X$ " vermelho desenhado na palma da mão, para que o atendente reconheça a pessoa como vítima de violência e acione as autoridades, facilitando a identificação dos casos.

Em 2003 foi promulgada a Lei nº 10.778 que estabelece a notificação compulsória, em território nacional, dos casos de violência contra mulher atendida em Serviços de Saúde públicos e privados. Sendo esta, uma das obrigações do enfermeiro perante $o$ atendimento a uma mulher vítima de violência (Brasil, 2003).

Na cidade de Recife/PE, existe uma Rede de Enfrentamento a Violência Doméstica e Familiar Contra a Mulher, que se apoia na notificação dos casos, assim como efetivo enfrentamento. A atenção básica, funciona como porta de entrada para esses serviços, como o Centro de Referência em Assistência Social (CRAS), Centro de Referência Especializado em Assistência Social (CREAS), Centro de Apoio Psicossocial (CAPS), Unidades de Saúde da Família (USF), Policlínicas, Hospitais, Unidades de Pronto Atendimento, Centro de Referência em Direitos Humanos, entre outros (Pernambuco, 2014).

Sendo assim, fica explícito que o ponto máximo dessa violência estrutural sistemática contra a mulher é o feminicídio, entendido como a consequência final de um ciclo de violência na qual a mulher está inserida. Ele pode ser classificado como uma morte evitável, pois há uma série de violências que são antecedentes e constituintes a ela, por isso, é necessário um olhar cuidadoso e criterioso para os antecedentes por parte dos profissionais de saúde (De Souza, 2018).

Existem alguns mecanismos que contribuem para a perpetuação da violência até o feminicídio, como: a tolerância social de algumas formas de violência contra mulher, a insuficiência de serviços públicos de atendimento, segurança e justiça, a negligência dos profissionais que atuam nesses serviços, a impunidade e até a proteção de autores de violências, por meio da culpabilização da mulher (De Souza, 2018). Perceber que muitas dessas mortes poderiam ser evitadas, revela uma conivência social e institucional, com aspectos culturais marcantes como o sentimento de posse associado ao crime cometido em relações íntimas de afeto. 
Observa-se também, que o Estado apresenta-se com poucos recursos destinados à prevenção da violência contra mulher. Falta ao poder público trabalhar com políticas de prevenção e conscientização, além de investir nos meios de apoio e inclusão de mulheres violentadas (Trigueiro, et al. 2015). Portanto, entende-se que para combater o feminicídio, é necessário intervir nas situações de violência cotidiana pela qual as mulheres passam. Para isso, é preciso que discussões sobre o tema sejam feitas desde a escola, como por exemplo, sobre desigualdade de gênero, machismo, misoginia e preconceitos, até ambientes abertos, como atividade educativa em comunidades, almejando assim, fornecer vias de conscientização da rede de apoio e denúncia à violência como forma preventiva da morte de mulheres.

O papel da enfermagem na prevenção ao feminicídio faz-se por meio do atendimento às vítimas de violência doméstica/intrafamiliar pregressa. Os profissionais devem estar atentos durante o atendimento, pois a maneira como é realizada a assistência potencializa as denúncias, retirando-as da condição de vulnerabilidade e efetiva as leis de proteção às mulheres (Santos, et al. 2018). A assistência de enfermagem para essas mulheres deve ser pensada para promover o acolhimento, o respeito, a segurança e a satisfação das suas necessidades individuais e coletivas (De Souza, 2018). Por isso, o planejamento deve ser pautado nos instrumentos básicos da enfermagem, nas políticas públicas de saúde e na legislação vigente, sendo fundamental para a proteção das vítimas e prevenção dos casos de violência e dos seus futuros agravos, como o feminicídio.

A primeira parte da abordagem aos casos de violência doméstica é a percepção por meio de sinais e sintomas de ordem física, emocional e sexual (Brasil, 2016). Alguns sinais e sintomas mais frequentes entre mulheres vítimas atendidas pela equipe de enfermagem no serviço são: afastamento, tristeza, abatimento, medo de ficar sozinha, distúrbios de sono, agressividade, dificuldade de concentração, desidratação, desnutrição, perda ou excesso de apetite. Ao exame físico, apresentam manchas, queimaduras, feridas, alopecia, intoxicação, lesão na área genital, infecções urinárias e vaginais, gravidez indesejada, entre outros.

O próximo passo após a identificação dos sinais e sintomas, é a conversa com a vítima e seus familiares a respeito da violência por meio da escuta qualificada. Será realizada uma avaliação global por meio de uma entrevista e um exame físico geral e específico. Um dos profissionais da equipe multidisciplinar irá realizar o acolhimento da paciente observando os princípios do respeito, da dignidade, da não-discriminação, do sigilo, e da privacidade, de forma a garantir um atendimento humanizado e seguro (Santos, et al. 2018). É importante que o profissional utilize um espaço reservado para a entrevista, além de demonstrar respeito para construir uma relação de confiança com essa mulher, além de não mostrar parcialidade em relação à situação e realizar as perguntas de forma aberta e direta (Brasil, 2016).

Depois de confirmado o caso de violência doméstica, é importante que o profissional faça as orientações à vítima e sua família de como será o prosseguimento do caso, além de instruir sobre a importância do registro da ocorrência para sua proteção e da sua família. A vítima deve dirigir-se ao centro de saúde ou hospital, para receber atendimento caso seja necessário (Pernambuco, 2010). O profissional deve informar a mulher que não deve ser feita a higiene pessoal, pois o banho pode comprometer a coleta de provas, principalmente nos casos de violência sexual, que devem constar no laudo pericial do IML (Pernambuco, 2010).

Todos os profissionais de saúde que se deparam com vítimas de violência devem realizar a notificação obrigatória a uma autoridade pública, para registro e classificação desta mulher perante as entidades públicas (Brasil, 2014). Essa notificação compulsória é de extrema necessidade para conhecer melhor a magnitude da violência, pois existem muitos dados subnotificados que inviabilizam e dificultam a realização de novos estudos sobre o tema. As notificações realizadas são encaminhadas da Unidade de Saúde para o setor de vigilância epidemiológica da Secretaria Municipal de Saúde (Pernambuco, 2014).

De acordo com o Protocolo Da Atenção Básica Para Saúde Da Mulher, elaborado pelo Ministério da Saúde em 2016, a postura do profissional durante o atendimento deve seguir alguns parâmetros, como a não vitimização das mulheres e a consciência crítica para lidar com as emoções que podem surgir durante o acompanhamento (Acosta, et al. 2017). É necessário 
que o profissional de enfermagem elabore perguntas diretas durante a anamnese, pois muitas mulheres não realizam a queixacrime e essa abordagem pode facilitar a identificação por meio de perguntas como: "Você já sentiu medo de alguém? Você se sente humilhada? Você acha que os problemas da sua casa estão afetando sua saúde?” (Brasil, 2016).

Durante a entrevista, o enfermeiro deverá detectar a situação de vulnerabilidade da mulher, identificando se a situação de violência é recorrente ou não por meio da percepção dos sinais de alerta: início tardio do pré-natal, parceiro controlador, infecção urinária de repetição, dor pélvica, aborto de repetição, complicação na gestação, depressão, ansiedade, transtorno de estresse pós-traumático, história de tentativa de suicídio, e lesões físicas (Brasil, 2016).

Por último, acontece o exame físico geral, que será realizado por um Enfermeiro, no qual é feito o exame preventivo de câncer do colo do útero e o teste para IST em casos de violência sexual (Santos, et al 2018). Deve-se atentar para recusa ou dificuldade para realização do exame. Observar se existe presença de ferimentos que não foram explicados e realizar inspeções detalhadas das partes do corpo que podem revelar sinais de violência como, tronco, membros, nádegas, cabeça e pescoço (Trigueiros, et al. 2015). Caso a usuária não relate a violência ou possua atendimento anteriores com sinais de violência, qualquer referência ao fato deve estabelecer a necessidade de atenção por parte da equipe.

O Enfermeiro precisa ter um olhar diferenciado ao trabalhar com vítimas de violência. Devido à falta de capacitação de alguns profissionais, o atendimento muitas vezes não é feito da forma correta e humanizada, necessária na atenção à saúde (Santos, et al. 2018). É preciso entender que existem limitações no preparo dos Enfermeiros para o atendimento das vítimas de violência, pois existe uma falta de estrutura de recursos humanos e escassez de materiais de capacitação para esses profissionais (Brasil, 2014).

A percepção dos Profissionais de Saúde em relação ao cuidado da mulher em situação de violência revela um distanciamento e evidente sofrimento em atender essa demanda de grande complexidade (Acosta, et al. 2017). Por isso, é necessário que os serviços ponham maior ênfase ao combate e prevenção da violência, possibilitando a melhoria da assistência prestada ao cliente. Para isso é importante a realização de capacitações sobre a violência contra a mulher, suas formas de prevenção, a promoção do acolhimento solidário e o encorajamento da notificação. Dessa forma, os Enfermeiros estariam cada vez mais capacitados a atender esse público, de forma a promover uma intervenção eficaz e humanizada capaz de restabelecer os direitos de autonomia, autoestima e independência da mulher vítima da violência doméstica/intrafamiliar, retirando-as desse ciclo violento evitando assim consequências fatais como o feminicídio (Santos, et al. 2018).

O tema proposto é bastante relevante no momento atual, em que percebe-se uma grande prevalência de casos de violência e feminicídio. Além disso, grande parte dos Enfermeiros apresentam um conhecimento defasado em relação à forma correta de percepção, notificação e assistência aos casos de violência contra a mulher. Por isso, este estudo tem por finalidade mostrar como os profissionais de enfermagem devem agir nesses casos, buscando retirar a vítima do ciclo violento evitando assim uma possível morte, e lhe proporcionando a retomada da sua autonomia e independência.

A pesquisa mostrou que o número de mulheres vítimas é crescente, assim como o número de notificações também está aumentando e isso se deve a uma melhor estratégia para denúncia. Resultados anteriores a 2006 demonstram subnotificação dos dados, devido a inexistência, até então, da Lei Maria da Penha. Outro fator relevante é o medo ainda presente nas vítimas de realizar a denúncia e procurar ajuda. Percebeu-se também que poucos estudos foram realizados para abordar o aspecto de transidentidade e raça dentro do tema da violência doméstica e intrafamiliar, embora os números de vítimas pretas e trans sejam os maiores entre as mulheres.

Foi percebido uma limitação em relação aos descritores, pois encontramos poucos artigos que correlacionaram a enfermagem e o feminicídio. Mudamos o enfoque e passamos a procurar como a enfermagem poderia atuar para redução dos casos de violência doméstica e consequentemente para a diminuição dos números de feminicídios. 


\section{Considerações Finais}

A partir de observações, diálogos e aplicação dos mecanismos de pesquisa, foram empregadas habilidades e competências desenvolvidas ao longo do período vigente, as quais mostraram-se fatores primordiais para analisar o papel da enfermagem na prevenção ao feminicídio. Dessa forma, o SUS tem extrema importância na problemática da violência contra mulher, uma vez que além do cuidado imediato aos danos físicos e emocionais, os Enfermeiros devem reconhecer o problema, acolher a vítima e referir para a rede especializada a fim de garantir os direitos das mulheres.

Nessa perspectiva, foi possível compreender que o papel da equipe de enfermagem é proporcionar um atendimento com ações humanizadas e eticamente seguras por meio de medidas de prevenção e tratamento contra os diversos tipos de violência, de acordo com as políticas públicas de saúde e da legislação vigente, sendo fundamental para a proteção das vítimas e prevenção dos casos de violência e dos seus futuros agravos, como o feminicídio.

Ao término do trabalho analisa-se que, apesar de ser um tema de total relevância na saúde pública atualmente, é preciso ampliar o debate acerca da violência de gênero dentro dos serviços de saúde para que, dessa forma, os profissionais de enfermagem possam compreender o fenômeno e lidar com as consequências da violência sofrida, sabendo que o cuidado prestado a essas mulheres é, em sua maioria, realizado por essa classe profissional.

\section{Referências}

Acosta, D. F. et al. (2017). Aspectos éticos e legais no cuidado de enfermagem às vítimas de violência doméstica. Texto contexto - enferm. 26 (3), e6770015.

Andrade, S. R. et al. (2017). O estudo de caso como método de pesquisa em enfermagem: uma revisão integrativa. Texto \& contexto enferm. $26(4)$, e5360016.

Arboit, J. et al. (2017). Health care for women in situations of violence: discoordination of network professionals. Rev. esc. enferm. USP 51 , e03207.

Benevides, B. G; Nogueira, s. N. B. (2021). Dossiê assassinatos e violência contra travestis e transexuais brasileiras em 2020. Orgs. São Paulo: Expressão Popular, ANTRA, IBTE <https://antrabrasil.files.wordpress.com/2021/01/dossie-trans-2021-29jan2021.pdf〉.

Brasil. (2021). Denunciar e buscar ajuda a vítimas de violência contra mulheres (Ligue 180). Brasil. <https://www.gov.br/pt-br/servicos/denunciar-e-buscarajuda-a-vitimas-de-violencia-contra-mulheres>.

Brasil. (2006). Lei nº 11.340. Brasil. http://www.planalto.gov.br/ccivil_03/_Ato2004-2006/2006/Lei/L11340.htm>.

Brasil. (2015). Lei no 13.104. Brasil. http://www.planalto.gov.br/ccivil_03/_ato2015-2018/2015/lei/113104.htm>.

Brasil. Ministério da Saúde. (2016). Protocolos da Atenção Básica: Saúde das Mulheres. Ministério da Saúde, Instituto Sírio-Libanês de Ensino e Pesquisa. Brasília/DF. <https://bvsms.saude.gov.br/bvs/publicacoes/protocolos_atencao_basica_saude_mulheres.pdf>.

Brasil. (2021). Sancionada lei do Sinal Vermelho contra violência doméstica. Brasil. <https://www.gov.br/pt-br/noticias/assistencia-social/2021/07/sancionadalei-do-sinal-vermelho-contra-violencia-domestica>.

Cardoso, F. S. Brito, L. M. T. (2015). Possíveis impasses da Lei Maria da Penha à convivência parental. Estudos e Pesquisas em Psicologia, 15 (2), 529-546.

Carrijo, C. Martins, P. A. (2020). A violência doméstica e racismo contra mulheres negras. Rev. Estud. Fem. $28(2)$, e60721.

Colimoide, F. P. et al. (2017). Integralidade na perspectiva de enfermeiros da Estratégia Saúde da Família. Rev. Bioét. 25 (3), $611-617$

De Sousa, L. M. M. et al. (2017). A metodologia de revisão integrativa da literatura em enfermagem. Revista Investigação em Enfermagem; $17-26$.

De Souza, S. M. J. (2018). O Feminicídio e a legislação brasileira. Rev. Katálysis $21 \quad$ (03). https://www.scielo.br/j/rk/a/XHsBp yL7bg56mBKqDpfQ88y/?lang=pt>.

Fórum Brasileiro de Segurança Pública - FBSP. Práticas de Enfrentamento à violência contra as mulheres: experiencias desenvolvidas pelos profissionais de segunrança pública e do sistema de justiça - Casoteca FBSP 2019. Fórum Brasileiro de Segurança Pública. São Paulo: Fórum Brasileiro de Segurança Pública, 2020. - (Série Casoteca FBSP, v.3). 216p. https://forumseguranca.org.br/wp-content/uploads/2021/02/casoteca-2019-v5.pdf>.

Honnef, F. et al. (2021). Representações sociais da violência doméstica em cenários rurais para mulheres e homens. Acta Paul Enferm. $368-374$.

Ipea. (2020). Atlas de Violência. Instituto de Pesquisa Econômica Aplicada - IPEA. <https://www.ipea.gov.br/atlasviolencia/download/24/atlas-da-violencia$2020>$.

Martins, T. V. et al. (2020). O efeito das microagressões raciais de gênero na saúde mental de mulheres negras. Ciênc. saúde coletiva, 25 (7), pp. 2793-2802

Moura, L. S. (2020). Acolhimento e acompanhamento de mulheres vítimas de violência sexual: proposta de ferramenta de apoio. Dissertação de mestrado. Site online. Curitiba - PR, Brasil. 2020. https://acervodigital.ufpr.br/bitstream/handle/1884/70109/R\%20-\%20D\%20-\%20LETICIA\%20DE\%20SOUZA\% 20MOURA.pdf?sequence $=1 \&$ isAllowed $=\mathrm{y}>$. 
Research, Society and Development, v. 10, n. 13, e471101321350, 2021

(CC BY 4.0) | ISSN 2525-3409 | DOI: http://dx.doi.org/10.33448/rsd-v10i13.21350

Netto, L. A. et al. (2018). Atuação da enfermagem na conservação da saúde de mulheres em situação de violência. reme - Revista Mineira de Enfermagem, 22:e1149 .

Okabayashi, N. Y. et al. (2020). Violência contra a mulher e feminicídio no Brasil - impacto do isolamento social pela COVID-19. Brazilian Journal of Health Review. 3(3).

Pernambuco. Secretaria de Saúde do Estado de Pernambuco. (2010). Orientações para o atendimento a vítimas de violência: guia para profissionais de saúde. Secretaria de Saúde do Estado de Pernambuco - Secretaria executiva de vigilância em saúde, 2010. Série Acidentes e Violências - $\mathrm{N}^{\circ} 2$. <https://ead.saude.pe.gov.br/pluginfile.php/3816/mod_folder/content/0/Orienta\%C3\%A7\%C3\%B5es\%20para\%20atendimento\%20a\%20vitimas\%20de\%20vi ol\%C3\%AAncia_PE\%202010.pdf?forcedownload=1

Pernambuco. (2014). Rede de Enfrentamento à Violência Doméstica e Familiar contra a Mulher da cidade do Recife. Ministério Público de Pernambuco. Recife/PE. https://www.mppe.mp.br/mppe/attachments/article/2861/Livro_Pesquisa_Mulher_10leve.pdf>.

Rabelo, D. P. et al. (2019). Incidência da Violência contra a Mulher e a Lei do Feminicídio. Revista Brasileira Interdisciplinar de Saúde. https://revistarebis.rebis.com.br/index.php/rebis/article/view/55/51.

Santos, S. C. et al. (2018). Violência Contra a Mulher: Como os profissionais da atenção primária à saúde estão enfrentando essa realidade? Revista Saúde e Pesquisa, 11(2), 359-368.

Silva, J. O. M. et al. (2021). Planejamento e implementação do curso Sexual Assault Nurse Examiner para o atendimento às vítimas de violência sexual: relato de experiência. Rev Esc Enferm USP ;55, e03739

Souza, M. A. R. (2020). Educação permanente em saúde: desenvolvimento de competências profissionais na atenção às mulheres em situação de violência. Dissertação de mestrado. Site online. Curitiba - PR, Brasil. 2020. https://acervodigital.ufpr.br/bitstream/handle/1884/69966/R\%20-\%20T\%20\%20MARLI\%20APARECIDA\%20ROCHA\%20DE\%20SOUZA.pdf?sequence=1\&isAllowed=y >

Trigueiro, T. H., et al. (2015). Vítimas de violência sexual atendidas em um serviço de referência. Ver. Cogitare Enferm. Abr/jun; 20(2):249-56.

Zabala, T. C. (2020). Violência Doméstica Contra a Mulher Transgênera e a Mulher Travesti. Revista Essência Jurídica - UniFCV. 3(1). 